\title{
Little groups and Maxwell-type tensors for massive and massless particles
}

\author{
S. BAŞKAL ${ }^{1}$ and Y. S. KIM ${ }^{2}$ \\ ${ }^{1}$ Department of Physics, Middle East Technical University - Ankara 06531, Turkey \\ 2 Department of Physics, University of Maryland - College Park, MD 20742, USA
}

(received 17 July 1997; accepted 30 September 1997)

PACS. 11.30Cp - Lorentz and Poincaré invariance.

PACS. 02.20Qs - General properties, structure, and representation of Lie groups.

PACS. 03.50De - Maxwell theory: general mathematical aspects.

\begin{abstract}
The massive and massless representations of four-vectors and Maxwell-type tensors are constructed as bilinear combinations of $S L(2, C)$-spinors and are evaluated on a unified description upon adopting the group contraction procedure of $O(3)$-like little group to $E(2)$-like little group. Contraction of massive particle representations leads to gauge-dependent vectors as well as the gauge-independent "state vectors" constructed by Weinberg. It is shown that gauge degrees of freedom associated with the translation-like transformations of the $E(2)$-like little group can be traced to the spinors that undergo spin flips in the infinite-momentum/zero-mass limit.
\end{abstract}

One of the beauties of Einstein's special relativity is the unified description of the energymomentum relation for massive and massless particles through $E=\left[(P c)^{2}+\left(M c^{2}\right)^{2}\right]^{1 / 2}$. In addition to mass, energy and momentum, relativistic particles have internal degrees of freedom, whose symmetries for massive and massless particles are dictated by $O(3)$-like and $E(2)$-like little groups, respectively [1]. It was shown by Inönü and Wigner that the rotation group $O(3)$ can be contracted to $E(2)$ [2]. The unified description of internal symmetries for both massive and massless particles can be obtained through the contraction procedure, identical to that of Inönü and Wigner, which in essence is taking the infinite-momentum/zero-mass limits of their massive counterparts [3].

In this article we are interested in constructing four-vectors and Maxwell-type tensors from spinors, in a unified scheme both for massive and massless particles, through adopting an analogous approach as that of the contraction procedure of the $O(3)$-like little group into $E(2)$ like little group. We will work within the framework of Wigner's little groups, which leave the four-momentum of a given particle invariant. In this context, we will conclude that the $E(2)$ like group transformations on the four-potential are equivalent to gauge transformations. It will also be shown that unlike the four-potential, which comprises both the parameter-dependent and -independent spinors, the electromagnetic-field tensor comprises only the parameterindependent spinors. The $S L(2, C)$ regime will prove to be appropriate for our purposes.

(C) Les Editions de Physique 
The group of Lorentz transformations is generated by three rotation generators $J_{i}$ and three boost generators $K_{i}$. They satisfy the commutation relations of the $S L(2, C)$ Lie algebra:

$$
\left[J_{i}, J_{j}\right]=i \epsilon_{i j k} J_{k}, \quad\left[J_{i}, K_{j}\right]=i \epsilon_{i j k} K_{k}, \quad\left[K_{i}, K_{j}\right]=-i \epsilon_{i j k} J_{k}
$$

The first solution of this set of commutation relations consists of $J_{i}=\frac{1}{2} \sigma_{i}, K_{i}=\frac{i}{2} \sigma_{i}$, and the second set consists of $J_{i}=\frac{1}{2} \sigma_{i}, \dot{K}_{i}=-\frac{i}{2} \sigma_{i}$. We call these two representations "undotted" and "dotted" representations, respectively. In addition to the usual normalized Pauli spinors $\alpha$ and $\beta$, for spin in the positive and negative $z$-directions respectively, there is another independent set of spinors $\dot{\alpha}$ and $\dot{\beta}$ associated with the dotted representation. These two sets of spinors have quite different Lorentz-boost properties.

The rotation matrix $R(\theta)=\exp \left[-i \theta J_{3}\right]$ is the same for both representations. The boost matrix in the undotted representation is

$$
B(\eta)=\exp \left[-i \eta K_{3}\right]=\left(\begin{array}{cc}
e^{\eta / 2} & 0 \\
0 & e^{-\eta / 2}
\end{array}\right)
$$

while it becomes

$$
\dot{B}(\eta)=\exp \left[-i \eta \dot{K}_{3}\right]=\left(\begin{array}{cc}
e^{-\eta / 2} & 0 \\
0 & e^{\eta / 2}
\end{array}\right)
$$

for the dotted representation.

For a massive point particle there is a Lorentz frame in which the particle is at rest. In this frame, the little group is clearly the three-dimensional rotation group $O(3)$. The fourmomentum is not affected by this rotation, but the spin variable changes its direction. Hence, the little group of a moving massive particle can be obtained by boosting along the direction of the momentum. Without loss of generality, if the particle is boosted in the $z$-direction, the generators of the little group can be obtained by

$$
J_{i}^{\prime}=B(\eta) J_{i} B(\eta)^{-1} .
$$

Since $J_{3}$ commutes with $K_{3}$ it remains invariant, while $J_{1}$ and $J_{2}$ assume the form

$$
J_{1}^{\prime}=\cosh \eta J_{1}+\sinh \eta K_{2}, \quad J_{2}^{\prime}=\cosh \eta J_{2}-\sinh \eta K_{1},
$$

which satisfy the Lie algebra of $O(3)$. Thus, the little group for a massive particle is a covariant entity and remains $O(3)$-like in all Lorentz frames.

However, for massless particles there are no Lorentz frames in which the particle is at rest. The approach is to consider the limiting case in which the mass of the particle becomes vanishingly small yielding the boost parameter to become infinite. After renormalizing the generators $J_{1}^{\prime}$ and $J_{2}^{\prime}$, in the infinite- $\eta$ limit they reduce to

$$
N_{1}=K_{1}-J_{2}, \quad N_{1}=K_{2}+J_{1} .
$$

The operators $N_{1}, N_{2}$ and $J_{3}$ satisfy the commutation relations

$$
\left[J_{3}, N_{1}\right]=i N_{2}, \quad\left[J_{3}, N_{2}\right]=-i N_{1}, \quad\left[N_{1}, N_{2}\right]=0,
$$

where $J_{3}$ is like the rotation generator, while $N_{1}$ and $N_{3}$ are like translation generators in the two-dimensional Euclidean plane. Hence, they are the generators of the $E(2)$-like little group for massless particles. 
The little groups for the undotted and dotted spinors are to be constructed accordingly. $N_{1}$ and $N_{2}$ applicable to $\alpha$ and $\beta$ become

$$
N_{1}=\left(\begin{array}{cc}
0 & i \\
0 & 0
\end{array}\right), \quad N_{2}=\left(\begin{array}{ll}
0 & 1 \\
0 & 0
\end{array}\right),
$$

while for $\dot{\alpha}$ and $\dot{\beta}$ one has to employ

$$
\dot{N}_{1}=\left(\begin{array}{cc}
0 & 0 \\
-i & 0
\end{array}\right), \quad \dot{N}_{2}=\left(\begin{array}{cc}
0 & 0 \\
1 & 0
\end{array}\right) .
$$

The transformation matrices assume the form

$$
D(u, v)=\exp \left[-i u N_{1}-i v N_{2}\right]=\left(\begin{array}{cc}
1 & u-i v \\
0 & 1
\end{array}\right),
$$

and

$$
\dot{D}(u, v)=\exp \left[-i u \dot{N}_{1}-i v \dot{N}_{2}\right]=\left(\begin{array}{cc}
1 & 0 \\
-u-i v & 1
\end{array}\right) .
$$

Two of the spinors are invariant under this transformation:

$$
D(u, v) \alpha=\alpha, \quad \dot{D}(u, v) \dot{\beta}=\dot{\beta},
$$

while the other two depend on the translation-like parameters and undergo spin flips:

$$
D(u, v) \beta=\beta+(u-i v) \alpha, \quad \dot{D}(u, v) \dot{\alpha}=\dot{\alpha}-(u+i v) \dot{\beta} .
$$

In a previous paper by one of us [4], it has been shown that spinors in (12) represent polarized neutrinos, which is a consequence of the requirement of invariance under translation-like parameters.

A four-vector $V^{\mu}=\left(V_{x}, V_{y}, V_{z}, V_{t}\right)$ representing a massive particle can be expressed in the form of a two-by-two Hermitian matrix by $V=\sum \sigma_{\mu} V^{\mu}$, or equally well by $\dot{V}=\sigma_{0} V_{t}-\sum \sigma_{i} V^{i}$, where $\sigma_{0}$ is the identity matrix. Under rotations and boosts it is transformed as

$$
V^{\prime}=R V R^{\dagger}=R V R^{-1}, \quad V^{\prime}=B V B^{\dagger}=B V B .
$$

As the notation suggests, $\dot{V}$ admits $\dot{B}$ as the transformation matrices for boosts. It is possible to shift from $V$ to $\dot{V}$ by a matrix conjugation. Let us introduce $g=i \sigma_{2}$. Since it commutes with $\sigma_{2}$ but anticommutes with $\sigma_{1}$ and $\sigma_{3}$, it satisfies $\sigma_{i}=-g \sigma_{i}^{\mathrm{T}} g^{-1}$. Therefore, we have

$$
\dot{V}=g V^{\mathrm{T}} g^{-1},
$$

which we call the " $g$-conjugation". The $g$-conjugation of the rotation matrix results in its Hermitian conjugate: $R^{\dagger}=R^{-1}=g R^{\mathrm{T}} g^{-1}$. The boost along an arbitrary direction takes the form $B(\eta)=R B_{3}(\eta) R^{\dagger}$. The $g$-conjugation of the boost matrix results in its inverse or dot-conjugation: $\dot{B}=B^{-1}=g B^{\mathrm{T}} g^{-1}$.

To construct a four-vector from $S L(2, C)$-spinors, the tensor product of one undotted and one dotted spinor is to be taken into account. For simplicity in the notation, when no confusion is possible $\alpha$ and $\dot{\alpha}$ are one if the spin is up, and are zero if the spin is down, while $\beta$ and $\dot{\beta}$ are zero and one for the spin-up and -down cases. Considering the transformation properties of these spinors, each component of the four-vector is identified as

$$
\begin{array}{rlrl}
V_{x} & \simeq \frac{1}{2}(-\alpha \dot{\alpha}+\beta \dot{\beta}), & V_{z} & \simeq \frac{1}{2}(\alpha \dot{\beta}+\dot{\alpha} \beta), \\
V_{y} & \simeq \frac{i}{2}(-\alpha \dot{\alpha}-\beta \dot{\beta}), \quad V_{t} \simeq \frac{1}{2}(\alpha \dot{\beta}-\dot{\alpha} \beta) .
\end{array}
$$


Furthermore, $g$ converts $\alpha$ to $\beta, \beta$ to $-\alpha$, and similarly, $\dot{\alpha}$ to $\dot{\beta}$ and $\dot{\beta}$ to $-\dot{\alpha}$. Then, the matrix $V$ can be expressed as

$$
V=\left(\begin{array}{ll}
\alpha \dot{\beta} & -\alpha \dot{\alpha} \\
\beta \dot{\beta} & -\beta \dot{\alpha}
\end{array}\right)=\left(\begin{array}{c}
\alpha \\
\beta
\end{array}\right)(\dot{\beta},-\dot{\alpha}) .
$$

The transformation matrix acting on $(\dot{\beta},-\dot{\alpha})$ from the right is the $g$-conjugation of the transformation matrix acting on $(\dot{\alpha}, \dot{\beta})^{\mathrm{T}}$ from the left. Therefore $(17)$ is viable, with a well-defined Lorentz transformation.

For the case of a massless particle let us consider a photon moving in the $z$-direction. The four-vector wave function takes the form $A^{\mu}(x)=A^{\mu} e^{i w(z-t)}$, where $A^{\mu}=\left(A_{x}, A_{y}, A_{z}, \varphi\right)$. The four-momentum vector is then $p^{\mu}=(0,0, w, w)$. $A^{\mu}$ can be written in terms of $S L(2, C)$ spinors by considering the massless counterpart of (16). First they are boosted in the $z$ direction

$$
V_{z}^{\prime} \simeq \frac{1}{2}\left(e^{\eta} \alpha \dot{\beta}+e^{-\eta} \dot{\alpha} \beta\right), \quad V_{t}^{\prime} \simeq \frac{1}{2}\left(e^{\eta} \alpha \dot{\beta}-e^{-\eta} \dot{\alpha} \beta\right),
$$

where $V_{x}$ and $V_{y}$ are invariant quantities. Then, the infinite limit of the boost parameter is taken, which in essence is an infinite-momentum/zero-mass limit. After renormalizing the larger components, $A^{\mu}$ is identified as

$$
A_{x} \simeq \frac{1}{2}(-\alpha \dot{\alpha}+\beta \dot{\beta}), \quad A_{y} \simeq \frac{i}{2}(-\alpha \dot{\alpha}-\beta \dot{\beta}), \quad A_{z}=\varphi \simeq \frac{1}{2} \alpha \dot{\beta} .
$$

This accounts for the fact that the consequence of the Lorenz gauge $\left(\partial_{\mu} A^{\mu}=0\right)$ in the four-vector representation of the potential is already inherent in its spinor representation. We see that the gauge-dependent four-potentials consist of both parameter-dependent and -independent spinors. Now, it is the $E(2)$-like little group, which is responsible for the transformation of the two-by-two matrix $A$

$$
A^{\prime}=D(u, v) A D(u, v)^{\dagger} .
$$

Explicitly, this is

$$
A^{\prime}=\left(\begin{array}{cc}
\varphi+A_{z}+u A_{x}+v A_{y} & A_{x}-i A_{y} \\
A_{x}+i A_{y} & 0
\end{array}\right)
$$

From the very construction of little groups the photon four-momentum is conserved, and therefore it is straightforward to see that the electromagnetic-field tensor derived from the transformed four-potentials (21) is left invariant. In view of this and (19) it can be concluded that the translational degrees of freedom of the $E(2)$-like little group can now be associated with the gauge degrees of freedom, and that the gauge dependence of $A^{\mu}$ originates from the "gauge-dependent" spinors appearing in (13). The relation between Lorentz and gauge transformations has been studied from different points of view by several authors [5]. Here we consider them in the $S L(2, C)$ regime and within the framework of little groups.

Consider an antisymmetric four-by-four matrix

$$
T=\left(\begin{array}{rrrr}
0 & -g_{z} & g_{y} & f_{x} \\
g_{z} & 0 & -g_{x} & f_{y} \\
-g_{y} & g_{x} & 0 & f_{z} \\
-f_{x} & -f_{y} & -f_{z} & 0
\end{array}\right) .
$$

It is well known that $f=\left(f_{x}, f_{y}, f_{z}\right)$ and $g=\left(g_{x}, g_{y}, g_{z}\right)$ transform like three-vectors under rotations. The four-by-four boost matrix is also well known for the above tensor. For our 
purposes, each component of the tensor $T$ is to be identified with spinor combinations having similar transformation properties. First note that $f_{z}$ and $g_{z}$ are invariant under boosts and under the rotation around the $z$-axis. The spinor combinations which satisfy these invariance requirements are $\frac{1}{\sqrt{2}}(\alpha \beta+\beta \alpha)$ and $\frac{1}{\sqrt{2}}(\dot{\alpha} \dot{\beta}+\dot{\beta} \dot{\alpha})$. The remaining components of $f$ and $g$ can be expressed in terms of the tensor product of two undotted and two dotted spinors:

$$
\left(\begin{array}{l}
f_{x} \\
f_{y} \\
g_{x} \\
g_{y}
\end{array}\right)=\frac{1}{4}\left(\begin{array}{rrrr}
1 & 1 & 1 & 1 \\
-i & i & -i & i \\
-i & -i & i & i \\
-1 & 1 & 1 & -1
\end{array}\right)\left(\begin{array}{c}
\beta \beta \\
\alpha \alpha \\
\dot{\beta} \dot{\beta} \\
\dot{\alpha} \dot{\alpha}
\end{array}\right)
$$

To realize the massless counterpart of the tensor $T,(23)$ is boosted in the $z$-direction to become

$$
\begin{array}{ll}
f_{x}^{\prime} \simeq \frac{1}{4}\left[e^{\eta}(\alpha \alpha+\dot{\beta} \dot{\beta})+e^{-\eta}(\beta \beta+\dot{\alpha} \dot{\alpha})\right], & g_{x}^{\prime} \simeq \frac{i}{4}\left[e^{\eta}(-\alpha \alpha+\dot{\beta} \dot{\beta})+e^{-\eta}(-\beta \beta+\dot{\alpha} \dot{\alpha})\right], \\
f_{y}^{\prime} \simeq \frac{i}{4}\left[e^{\eta}(\alpha \alpha-\dot{\beta} \dot{\beta})+e^{-\eta}(-\beta \beta+\dot{\alpha} \dot{\alpha})\right], & g_{y}^{\prime} \simeq \frac{1}{4}\left[e^{\eta}(\alpha \alpha+\dot{\beta} \dot{\beta})-e^{-\eta}(\beta \beta+\dot{\alpha} \dot{\alpha})\right] .
\end{array}
$$

Following the contraction procedure, $f^{\prime}$ and $g^{\prime}$ now represent the electric- and magnetic-field amplitudes, $E$ and $B$, where

$$
\begin{array}{ll}
E_{x} \simeq \frac{1}{4}(\alpha \alpha+\dot{\beta} \dot{\beta}), & B_{x} \simeq \frac{i}{4}(-\alpha \alpha+\dot{\beta} \dot{\beta}), \\
E_{y} \simeq \frac{i}{4}(\alpha \alpha-\dot{\beta} \dot{\beta}), & B_{y} \simeq \frac{1}{4}(\alpha \alpha+\dot{\beta} \dot{\beta}),
\end{array}
$$

and hence $E_{x}=B_{y}$ and $E_{y}=-B_{x}$, which manifest the properties of electromagnetic plane waves for a circularly polarized free photon propagating in the $z$-direction. This expression is only for the field propagating along the $z$-direction, however it can be rotated to suit an arbitrary direction. It is important to note that the second-rank tensor $T$ with (24) is quite different from the above expression of $F$. The tensor $T$ is for a massive particle and forms a representation space for the $O(3)$-like little group. For massless particles the only "gaugeinvariant" products are $\alpha \alpha$ and $\dot{\beta} \dot{\beta}$, and, therefore, only these products are accommodated as the components of the Maxwell tensor $F$.

The construction of vectors and tensors for massive particles as tensor products of $S L(2, C)$-spinors are well-established in the literature [6]. In this article they are evaluated on a unified description both for massive and massless particles. The physical interpretation of the translational degrees of freedom of the $E(2)$-like group as being responsible for the gauge degrees of freedom in electromagnetic potentials is elaborated. The four-vector representation of this problem has been studied by one us through four-by-four realizations of little groups [7]. Here, this problem is examined by considering the two-by-two Hermitian representation of the four-vector. Indeed, these gauge degrees of freedom emanate from the contraction of the transverse components of the rotation generators during the contraction process of the $O(3)$ like little group to the $E(2)$-like little group. It is also demonstrated that an antisymmetric tensor representing a massive particle becomes the Maxwell tensor for massless particles with spin-1 through the contraction procedure. In this context the electromagnetic theory for a massless free photon with definite helicity can be regarded as the limit of a free-field theory of massive spin-1 particles. This is a question of physical interest, since there are experimental results on the upper limit of the possible photon mass [8]. The approach we adopt naturally reveals the "state vectors" introduced by Weinberg [9] by resolving the issue in the absence of 
"gauge-dependent" spinors in the Maxwell tensor. He constructed these from gauge-invariant spinors to represent electric and magnetic fields by imposing subsidiary invariance conditions $((2.25)$ in $[9])$.

One of the authors (SB) would like to acknowledge the kind hospitality of the elementary particles group in the University of Maryland where this work has been done.

\section{REFERENCES}

[1] Wigner E. P., Ann. Math., 40 (1939) 149; Bargmann V. and Wigner E. P., Proc. Natl. Acad. Sci. (USA), 34 (1948) 211.

[2] Inönü E. and Wigner E. P., Proc. Natl. Acad. Sci. (USA), 39 (1953) 510.

[3] Misra S. P. and Maharana J., Phys. Rev. D, 14 (1976) 133; Ferrara S. and Savoy C., in Supergravity 1981, edited by S. Ferrara and J. G. TAYlOR (Cambridge University Press, Cambridge) 1982, p. 151; Han D., Kim Y. S. and Son D., J. Math. Phys., 27 (1986) 2228; Kwon B. and Villasante M., J. Math. Phys., 29 (1988) 560 and 30 (1989) 201.

[4] Han D., Kim Y. S. and Son D., Phys. Rev. D, 26 (1982) 3717.

[5] Janner A. and Jenssen T., Physica, 53 (1971) 1; 60 (1972) 292; Kuperzstych J., Nuovo Cimento B, 31 (1976) 1; HAN D. and KIM Y. S., Am. J. Phys., 49 (1981) 348; van Der BiJ J. J., van Dam H. and Ng Y. J., Physica A, 116 (1982) 307.

[6] Penrose R. and Rindler W., Spinors and Space-time (Cambridge University Press, Cambridge) 1984; Kim Y. S. and Noz M. E., Theory and Applications of the Poincaré Group (Reidel, Dordrecht) 1986.

[7] Kim Y. S. and Wigner E. P., J. Math. Phys., 28 (1987) 1175; 31 (1990) 55.

[8] Goldhaber A. S. and Nieto M. M., Rev. Mod. Phys., 43 (1971) 27.

[9] Weinberg S., Phys. Rev. B, 134 (1964) 882. 